\title{
Technology Support for Smart Computational Grid (SCG) in Future Wireless Systems
}

\author{
Johnson O. V. ${ }^{1} \quad$ Jinadu O.T. ${ }^{2}$ \\ 1.Department of Computer Science Department, Federal Polytechnic, Ile-Oluji, Nigeria \\ 2.Department of Computer Science Department, Rufus Giwa Polytechnic, Owo, Nigeria
}

\begin{abstract}
The communication grid is an emerging infrastructure that will fundamentally change the way we think - and use - computing. Communication grid is used by analogy with the electric power grid, as an infrastructure that provides pervasive access to electricity. Like the computer and a small number of other advances had a dramatic impact on human capabilities and utilization of the scarce frequency resource, it is believed that allowing all components of our information technology infrastructure - computational capabilities, databases, sensors, and people - to be shared flexibly as true collaborative tools, the Smart Computational Grid (SCG) will have a similar transforming effect, allowing new classes of applications to emerge. Improvement in spectrum usage achieved by CR technology and associated collaborative computations on white spaces. Every spectrum hole includes several channels. Implementing collaborative algorithms over the channels help track changing environment parameters and managed to achieve smooth handoffs, which is maximized to offer services ranging from low-data-rate non-reatime applications to high-speed real-time multimedia applications. With virtualization, the heterogeneous wireless access networks complement each other to deliver maximal signal strength, wider coverage, efficient data rate, low latency and reduced loss rate.
\end{abstract}

Keywords: computation, detection, grid, sensing, virtualization

DOI: $10.7176 / \mathrm{CEIS} / 10-7-04$

Publication date: November 30th 2019

\section{Introduction}

Grid Computing means that computing power and resources can be obtained as utility similar to

electricity - the user can simply request information and computations and have them delivered to him without necessity to care where the data he requires resides or which computer is processing his request (Goyal and Lawande, 2005). From the technical perspective Grid Computing means the virtualization and sharing of available computing and data resources among different organizational and physical domains. By means of virtualization and support for sharing of resources, scattered computing resources are abstracted from the physical location and their specific features and provided to the users as a single resource that is automatically allocated to their computing needs and processes.

At the core of Grid Computing therefore, are virtualization and virtual centralization as well as availability of heterogeneous and distributed resources based on collaboration among and sharing of existing infrastructures from different organizational domains which together build the Computing Grid. A user terminal can join in a cognitive network if it has wireless communication, networking and cognitive radio capability. The users of such network are classified as primary (licensed) and secondary (unlicensed) users. The secondary users are also called cognitive terminals (CT) or unlicensed because on fixed spectrum is used for their communication and every spectrum hole includes several channels (Akyildiz et al, 2006) and (Zhou et al, 2009). Each channel is used for communication when it is associated to a radio interface of some other nodes or terminals.

At present, business organizations have to survive and develop competitive advantage in a dynamic and turbulent environment of global competition and rapid business change. As they are under constant pressure to simultaneously grow revenue and market share while reducing costs on IT applications, they have been changing techniques. Three major trends can be observed as a result of the pressing needs to have impact on organizational requirements upon Information Technology (IT) support by: (i) striving towards high agility' (ii) ensuring globalization of activities to be able to take advantage of opportunities provided by a global economy, and (iii) targeting increased mobility.In dynamic business environments, agility is considered the key success factor for companies. Only companies with high agility can be successful in today's rapidly changing business environments. In literature, there are various definitions for the term agility: from general ones as for example, the ability of firms to sense environmental change and respond readily to business requirement is a factor of resource sharing over a pooled architecture.

Coming under the Institute of Electrical Electronics Engineers (IEEE) 802.22 wireless regional networks (WRAN) standard, Raman and Singh (2014) asserts that CR technology supports dynamic spectrum access (DSA) and boost up spectrum availability and utilization significantly. The architecture modifies the wireless systems to facilitate interoperability; mitigate interference and maximize throughput.

Typically, heterogeneous wireless systems are a form of wireless communication in which a transceiver can 
intelligently detect communication channels where all network entities have the same right to access the spectrum. Multiple access is allowed for coexistence and communication using the same portion of spectrum.

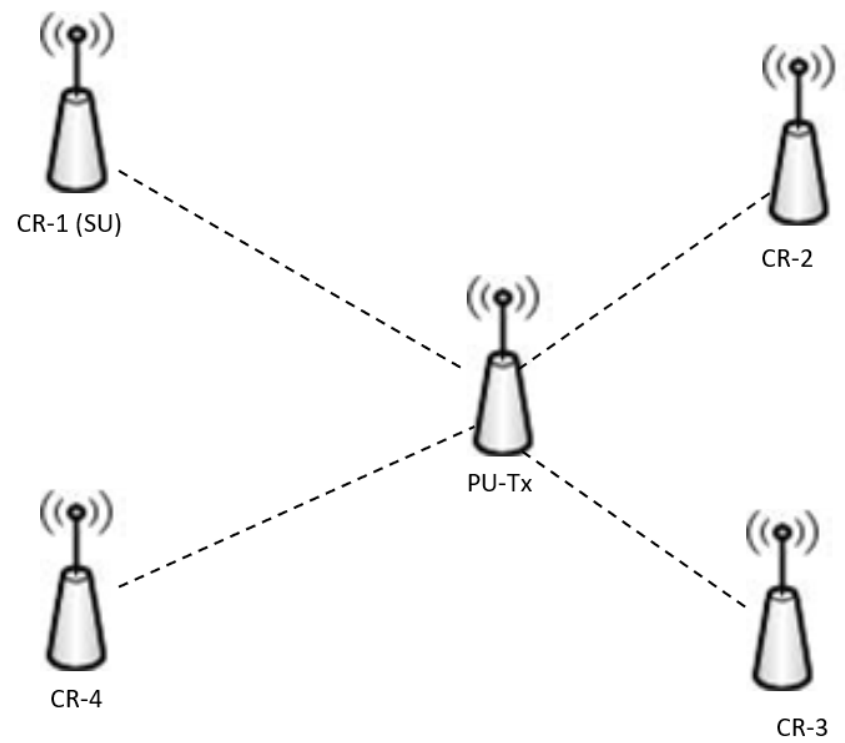

Fig. 1 Multiple Access Points (APs) centrally architecture

Similar to a grid network, the CR technology enables transmission parameters of many wireless nodes within its network to be changed to communicate while avoiding interference with original users. Therefore, configuration in cognitive networks became full reconfigurable system that automatically varies its communication variables with network and user demands (Ghosh, Das and Chatterjee, 2014). Technically, each communicating terminal is expected to be characterized and equipped with varied technologies and standards (IEEE 802.11*, 802.15*, 802.16*, 802.22 and $\mathrm{xG}$ ) and the interfaces includes UMTS, W-LAN/Wi-Fi, Wi-MAX, WSN, WRAN and others. Cognitive Radio Networking was thought of as an ideal goal of providing highly reliable communications whenever and wherever needed for efficient radio spectrum utilization. Hence the main goal of spectrum sensing to determine spectrum status and licensed user's activity is achievable with co-operative sensing as a form of distributed sensing. Provisioning for co-existence of heterogeneous devices operating in the spectrum holes is a drive for need to collaborate the sensing required to detect PU signals. Also, limited availability of spectrum and the non-efficient use of existing RF resources necessitate a new communication paradigm to exploit wireless spectrum opportunistically and even with greater efficiency (Mauri et al, 2014).

In view of the above, this paper is geared towards the objective of:

- modeling a framework for computation;

- collaboration for mutual parameter estimation; and

- intersystem handoff procedure to provide ubiquitous coverage in future systems

\section{Review on grid computing}

Spectrum management functionalities, which are in distributed co-ordination of CRN includes spectrum sensing, spectrum sharing, spectrum decision and spectrum mobility (Salem, AbdElKadir, Ramadan and Abdel -Mageed, 2014). The three basic types of spectrum sensing techniques for detecting PU licensed spectrum band outlined in Mauri et al (2014), therefore include co-operative, transmitter and interference-based spectrum sensing techniques. Cooperative detection allows multiple CRs to work together and supply required information to detect a PU. Spatial diversity intrinsic to multiuser network is exploited in distributed or centralized structure. Distributed approach enables CRs exchange spectrum observations among themselves while each develops a spectrum occupancy map based on signal information detected. This approach mitigates multipath fading and shadowing RF problems to increase probability of PU detection and also help combat common hidden node problem peculiar to ad hoc wireless networks.

The optimal detector in stationary Gaussian noise of communication channel is the matched filter, which maximizes received SNR of transmitted signals (frames). Matched filter technique of energy detection works by correlating known signal template with unknown signal and detecting presence of template in the unknown signal. Less time (frame duration) is required to achieve high processing gain due to coherency. Unlike other signaling techniques, (cyclo-stationary and interference-based) with long sensing time and complex computational tasks, matched filter detection only requires a prior knowledge of PU signal. This is readily made available in real-world situation as CR user monitors radio spectrum periodically to opportunistically communicate over spectrum holes. 


\section{Spectrum Sharing and DSA}

With spectrum sensing as first step in realizing cognitive radio communications, overview of MAC layer sensing issues and MAC protocol performance were outlined in Bayhan and Alagoz (2012). An enhancement for analyzed MAC scheme was also proposed as basis for a channel to forming cluster of sub-channels. The clustered approach enables the secondary nodes clustered into groups and each SU contends with the elements in the same cluster. This arrangement form set of sub-logical channels instead of a single logical channel.

PHY layer sensing accuracy highly depends on the sensing duration and in CR system, authorized or licensed user has the priority to occupy the frequency band, while the cognitive users (SUs) may only use the spectrum opportunistically. CRN enabled SUs utilize spectrum holes detected in licensed bands for their communication needs. Spectrum holes are free of PU existence or are partially occupied by low-power interferes.

CR operating parameters includes normalized transmission power, modulation scheme, transmission bit rate and centre frequency [sub-carrier bandwidth]. These are adjusted in order to operate in the dynamic spectrum usage required by next-generation heterogeneous wireless environments (Raman and Singh, 2014). Since static allocation of the frequency spectrum does not meet the needs of growing wireless technology, CR technologies offer efficient spectrum sharing through dynamic access (Chowdhury et al, 2012) and so DSA technique is capable of addressing spectrum scarcity problems. DSA as new spectrum sharing paradigm, allows SUs access to abundant spectrum holes in licensed bands (Song, Xin, Zhao and Cheng, 2012).

The reliability of cognitive operation depends on effective spectrum sensing as remarked in Raman and Singh (2014). Song et al earlier observed the need for new DSA model where licensed users would co-operate to provide much more flexible spectrum sharing. Also, to further solve the problem of spectrum scarcity, Ejaz et al (2012) described energy detection scheme as simple strategy with poor performance under low SNR.

\section{Threshold Setting in Signal Detection}

PHY-layer sensing adapts modulation schemes and parameters to measure and detect the primary users' signals on different channels. PHY-layer detection methods - energy detection, matched filter and feature detection are commonly used with feature detection being a good candidate for detecting PU's presence on channel as energy detection method. Detection results are always given in the following three possibilities of: channel is idle; channel is occupied by its primary users, but secondary users are allowed to transmit their packets with some power constraints; or channel is not available to secondary users at all. On the other hand, the MAC-layer sensing determines when and how a SU node sense and which channel(s) it senses (Firooz, Chen and Liu, 2013).

Two major threshold setting scheme in literature include probability of detection $\left(P_{d}\right)$ and probability of false alarm $\left(P_{f}\right)$, which is usually fixed to a small value (minimized) while $P_{d}$ is allowed to a big value (maximized). To maximize $P_{d}$ requires a constant false alarm rate (CFAR) principle. Similarly, to minimize $P_{f}$ while guaranteeing a non-interference probability, miss detection probability $\left(P_{m}\right)$ is set to a minimum and $P_{d}$ to a high value, the approach is called constant detection rate (CDR) principle.

\section{Detection Modeling}

Generally, received signal $y$ over CR model is expressed as

$$
y=h x+z y
$$

where $x$ is the transmitted symbol and $z$ the additive noise sample at receiver with $h$ being channel loss between source and destination. Using PU detection model, hypothesis test give $H_{0}$ to indicate only noise and $H_{1}$ to indicate PU presence. With noise signal s(n) assumed as stationary, independent, identical distributed random process of zero mean (AWGN) with power spectral density $N_{0}$ and variance $\sigma_{n}{ }^{2}$, detection performance is measured by probability of detection $\left(P_{d}\right)$ and probability of false alarm $\left(P_{f}\right)$.

$P_{d}$ indicates PU existence while $P_{f}$ indicates no PU in reality. Mathematically,

$$
\begin{aligned}
& P_{d}=\operatorname{Pr}\left(\text { signal }- \text { det ected } \mid H_{1}\right) \\
& P_{f}=\operatorname{Pr}\left(\text { signal }- \text { det ected } \mid H_{0}\right)
\end{aligned}
$$

Thus, $P_{d}$ should be big to protect the PU from interference and $P_{f}$ should be very small to increase spectrum usage efficiency. Minimum detectable signal level and sensing time is needed to achieve desired probabilities of detection and false alarm in AWGN. Also, with a matched filter implementation as digital domain, Cabric, Tkachenko and Brodersen (2010) theoretically obtained an optimal hypothesis testing solution for 
detection performance and it was measured by a pair of $\left(P_{d}, P_{f}\right)$ since $P_{f}$ denote false algorithm deciding PU present in scanned band when actually it is absent.

Stated in Bayhan and Alagoz (2012) and Raman and Singh (2014), PHY layer energy detection expression for the signal received by $\mathrm{SU}$ is given as

$$
x(n)= \begin{cases}w(n) & H_{0} \\ s(n)+w(n) & H_{1}\end{cases}
$$

where $n=1, \ldots, N$; and $\mathrm{N}$ is observation interval, $x(n)$ the signal received by $\mathrm{CR}, s(n)$ the PU transmitted signal and $w(n)$ the additive white Gaussian noise (AWGN) signal. Bayhan and Alagoz (2012) also presented how the PHY layer sensing statistically enables MAC sensing as depicted in fig. 2.

Common detection performance criteria, Neyman-Pearson likelihood ratio measured performance by a resulting pair of detection and false alarm probabilities $\left(P_{d}, P_{f}\right)$. Each pair is associated with the particular threshold $\gamma$ that tests the decision statistics:

$T>\gamma$ decide signal present; $T<\gamma$ decide signal absent

$P_{d}$ and $P_{f}$ can be evaluated simultaneously with

$$
P_{f}=Q\left(\frac{\gamma}{\sqrt{\varepsilon \sigma_{n}^{2}}}\right)
$$

and

$$
P_{d}=Q\left(\frac{\gamma-\varepsilon}{\sqrt{\varepsilon \sigma_{n}^{2}}}\right)
$$

Where the number of sample signal used in sensing is unlimited (scalability offered by cognition), energy detector provides for $P_{d}$ and $P_{f}$ simultaneously. The minimum number of sample signal subjected for detection is a therefore function of SNR evaluated and is given as

$$
S N R=\frac{\theta}{\sigma_{n}^{2}}
$$

and

$$
N=\left[Q^{-1}\left(p_{f}\right)-Q^{-1}\left(p_{d}\right)\right]^{2} S N R^{-1}
$$

where $\theta$ is the transmitted power

\section{Framework for collaborative computation}

Each wireless terminal is enabled to coexist with each other while being able to select a good channel. Though, characterized with intra-multiple user co-operation or competition collision challenge, the super-layer accurate sensing is guided against interference constraints. As depicted in fig. 2, PHY layer depend on sensing duration of other user detecting available spectrum opportunity locally whereas MAC sensing depends on sensing period and search order, but network layer while the network layer sensing is depicted by the SU co-operation.

Communicating channels are sensed using an estimation of channel occupancy probabilities as ordered by Latin square. To enable the multiple users maintain collision-free sensing, minimal false alarm is desired and each node is equipped with a cognitive radio to offer cogntion and reconfiguration techniques. 


\begin{tabular}{|c|c|c|c|c|c|}
\hline \multirow{3}{*}{$\begin{array}{l}\text { CR-1 sensing order } \\
\text { CR-2 sensing order } \\
\text { CR-3 sensing order }\end{array}$} & 1 & 2 & 3 & 5 & 4 \\
\hline & 81 & 2 & 3 & 5 & 4 \\
\hline & 2 & 4 & 5 & 3 & 1 \\
\hline
\end{tabular}

(a) CR-2 generate false alarm to avoid collision with CR-1

\begin{tabular}{l|l|l|l|l|l|}
\hline CR-1 sensing order & \multicolumn{1}{|l|}{2} & 3 & 4 & 5 \\
\cline { 2 - 6 } CR-2 sensing order & 3 & 4 & 5 & 1 \\
CR-3 sensing order & 5 & 1 & 4 & 2 & 2 \\
\cline { 2 - 7 } &
\end{tabular}

(b) CR-1 find channel 1 free in step 1

\begin{tabular}{|c|c|c|c|c|c|}
\hline \multirow{2}{*}{$\begin{array}{l}\text { CR-1 sensing order } \\
\text { CR-2 sensing order }\end{array}$} & 2 & $\$$ & 3 & 4 & 5 \\
\hline & 2 & 4 & 3 & 4 & 8 \\
\hline CR-3 sensing order & 3 & 14 & 5 & 1 & 2 \\
\hline
\end{tabular}

(c) CR-1 and 2 collide in step 2 (using same sensing order)

Key:

\begin{tabular}{|l|l|l|l|l|}
\hline $\begin{array}{l}\text { Occupied } \\
\text { by PU }\end{array}$ & $\begin{array}{l}\text { Two or } \\
\text { more CRs } \\
\text { find } \\
\text { channel } \\
\text { free }\end{array}$ & $\begin{array}{l}\text { Only one } \\
\text { CR find } \\
\text { channel } \\
\text { free }\end{array}$ & $\begin{array}{l}\text { False } \\
\text { alarm }\end{array}$ & $\begin{array}{l}\text { Busy } \\
\text { status }\end{array}$ \\
\\
\hline
\end{tabular}

Fig.3 Three CRs sensing three channels within same sensing duration

In scenario (a) and (c), CR-1 and CR-2 chose the same sensing order but collision was avoided by CR-1 in (a) by finding channel 1 free in step 1 while CR-2 generate a false alarm in that step. CR-1 and CR-3 find channels 3 and 1 free in steps 4 and 5. In scenario (b), only CR-1 find channel 1 free in step 1 because the same sensing order was not used by CR-1 and CR-2. CR-2 find channel 2 free in step 4 while finding channels 1 and 4 CR-3 find channel 2 free in step 3.

In scenario (c), CR-1 and CR-2 both find channel 4 busy in step 4 because of using same sensing order although this is resolved by CR-3 detecting non-existence of PU signal $\left(\mathrm{H}_{0}\right)$ in channel 4 (step 2) while CR-1 and CR-2 still find channel 5 busy. The same sensing order for two or more CRs is prevented to arrive at collisionfree detection of spectrum holes. From the framework, entire frequency band (licensed) have its allocated channels occupied (busy) mostly at every step servicing either PU or SU.

\section{Implementing Slotted Rendezvous for Collaboration}

Major objective of many CRs in the scenario is to offer intelligence and fair spectrum sharing to achieve collaboration and enhance the performance of the SCG while implementing radio rendezvous processes. As channels are sensed with highest probability being idle channel sensed first (CR-1 sensing channel 1,2,3,4,5 in scenario (b) while CR-2 in step 1 senses channel 3 first being the channel with highest probability of being idle since CR-1 had detected PU signal on it in step 3. With CR-1 and CR-2 sensing with the same order in (a) and (c), a rendezvous of obtained.

An ability of two or more radios meeting to establish a link on same channel provides for bootstrapped communication requirement of multi-channel systems. With this arrangement, SU is adversely affected in its sensing as the link maintains channel availability (DaSilva, 2012). Need for increased co-ordination between CR nodes is desired for detected available frequency bands (holes) to be joined together to from sub-channel logical band for SU access. A proactive plan to combat changing environmental conditions requires major task of detection probability accuracy.

While targeting how to maximize $P_{d}$ or minimize $P_{f}$, an optimized CFAR and CDR is evidently provided by the slotted rendezvous, which dynamically allows all channels $\{1,2, \ldots, N\}$ to be visited by radios in random, then in prescribed order scenario (b) and (c). Here, collaborative sensing by CRs increases system throughput since time to rendezvous (TTR) increases with N. Although, slotted rendezvous is equally likely to happen in any of the $\mathrm{N}$ channels, real-time sensing in search of white spaces maximizes TTR. This is a function of SNR of the signal samples. CRs multiplicatively decrease the probability of using same sensing order for two or more CRs. Frame duration comprises time slot for sensing, searching and transmission 


\begin{tabular}{|l|l|l|}
\hline Sensing Time Ts & Channel Search Tc & Transmission Tt \\
\hline
\end{tabular}

In agreement with Li, Liu and Roy (2012), each access frame has a fixed duration divided into two parts sensing slot and packet transmission slot. The sensing slot comprised of sensing time and channel search. Therefore, frame duration is a combination of sensing time $\left(T_{s}\right)$, channel search $\left(T_{c}\right)$ and Transmission $\left(T_{t}\right)$, the efficiency of spectrum usage for any typical sensing duration is expressed as

$$
\eta=\frac{T_{t}}{\left(T_{s}+T_{c}+T_{t}\right)}
$$

\section{Discussion}

To have a constant desired $P_{d}$ in dynamic scenario, number of samples can be varied for lower noise levels. The theoretical analysis shows that coherent processing can turn low SNR into high SNR regime so that, given enough samples, arbitrary weak signals can be detected. Scenarios (a) and (c) demonstrates performance of collaborative spectrum sensing in sequential order while scenario (b) showed sensing in random and then sequential order. When environment parameters change, SNR values changes $P_{f}$, which in effect changes for desired $P_{d}$.

The dynamic environment evident in SCG, higher $P_{f}$ is obtainable for small changes in $P_{d}$, which is one of the major metrics of measuring performance. Different values of $P_{d}$ can be obtained and evaluated for different SNR and channel selection based on incumbent appearance expectations in further research. Traditional channel selection mechanism could also be enhanced as well.

\section{Conclusion}

Consumption power, throughput, error rate and interference performance objective metrics of SCG has greater dependence on minimization of power consumption, which is directly placed on transmission power $(P)$ in agreement with Chen and Wyglinski (2009). Improved sensing capability is devised to track all changing environment condition as implemented in the computation. This connote that minimum detectable signal level and sensing time is required to achieve desired $P_{d}$ and $P_{f}$. Higher $P_{d}$ implies reduced $P_{f}$, and this increases CR$\mathrm{SU}$ exploitation of the unused spectrum adaptively in the radio environment.

Improvement offered by number of collaborative access point (AP) radios and threshold rule offer remarkable improvement in spectrum usage efficiency. This is further guaranteed with multiple cognitive terminal cooperating to maximize frame duration than individual terminal computing. Real-time sensing is offered by the framework in search of white spaces. This enable the SCG benefits tremendously from a proactive plan especially with changing noise variance along communication channels.

\section{References}

Akyildiz I.F., Lee W.Y., Vuran M.C. and Mohanty S.(2006). NeXt generation dynamic spectrum access cognitive radio wireless networks: a survey, Computer Networks Journal, Elsevier, 50 (13):2127-2159.

Bayhan S. and Alagoz F. (2012). MAC Layer Spectrum Sensing. Research by Women of Computing, SAT LAB, Bogazigi University, Turkiye.

Cabri D., Mishra S.M. and Brodersen R.M., "Implementation Issues in Spectrum Sensing”, Asilomar Conference on Signal, Systems and Computers, November 2004.

Chen S. and Wyglinski A.M.(2009). "Efficient Spectrum Utilization via cross-layer Optimization in Distributed Cognitive Radio Networks" Computer Communications, Elsevier Publishers

Chowdhury M., Kader A.M. and Raman O.M.(2012). Design of an Efficient MAC protocol for Opportunistic Cognitive radio Nteworks. International Journal of Computer Science \& Information Technology. Vol. 4. No. 5 October, 2012

Ejaz W., Hasan N. and Kim H.S. (2012), SNR-Based Adaptive Spectrum Sensing for Cognitive Radio Networks. International Journal of Innovative Computing, Information and Control, Vol 8, Number 9, September 2012.

Foster and Kesselman (2004)

Ghasemi A. and Sousa E.S, "Collaborative Spectrum Sensing for Opportunistic Access in Fading Environments", In proc. of DySPAN'05, November 2005.

Ghosh G., Das P. and Chatterjee S. (2014). "Cognitive Radio and Dynamic Spectrum Access - A study". 
International Journal of Next-Generation Networks. Vol.6 No.1, March, 2014.

Goyal and Lawande (2005).

Firooz M.H., Chen Z., Roy S. and Liu H.(2013). "Wireless Network Coding via Modified 802.11 MAC/PHY: Design and Implementation on SDR" IEEE International Conference on Communications (ICC.)

DaSilva L.(2012). Cognitive Networks: Theory and Practice of Cognitive Radios. Aalborg University, VirginiaTech. May 9-11, 2012.

Mauri J.L., Ghafoor K.Z., Rawat D.B., Samuel J. and Perez A.(2014), Cognitive Networks: Application and Deployments. Technology and Engineering Google Books, $1^{\text {st }}$ Ed. CRC Press Amazon.

Raman S. and Singh N.P.(2014). An Algorithm for Spectrum Sensing in Cognitive Radio under Noise uncertainty. International Journal of Future Generation Communication and Networking. Vol. 7. No. 3. 2014

Salem T.M, AbdElkader S.M., Ramadan .M. \& AbdelMageed M.Z.(2014). Opportunistic Spectrum Access in Cognitive radio AdHoc Networks. International Journal of Computer Science Issues Vol.11 Issue 1 No.2 January, 2014.

Song M., Xin C., Zhao Y. and Cheng X.(2012). Dynamic Spectrum Access: from Cognitive Radio to Network Radio. IEEE Wireless Communications. Vol 19 Issue 1.

Yadav P., Chatterjee S. and Bhattacharya P. (2014). "A Survey on Dynmic Spectrum Access Techniques in Cognitive Radio". International Journal of Next-Generation Networks, Vol.4, No.4, December, 2012. 